\title{
AERODYNAMIC CHARACTERISTICS OF AIRFOIL USING PANEL METHOD
}

\author{
Buyung Junaidin \\ Prodi Teknik Penerbangan \\ Sekolah Tinggi Teknologi Adisutjipto \\ Jalan Janti Blok-R Lanud Adisutjipto, Yogyakarta \\ ikh_libi_dikh@yahoo.com
}

\begin{abstract}
Potential flow over an airfoil plays an important historical role in the theory of flight. The governing equation for potential flow is Laplace's equation, a widely studied linear partial differential equation. One of Green's identities can be used to write a solution to Laplace's equation as a boundary integral. Numerical models based on this approach are known as panel methods in the aerodynamics community. This paper introduces the availability of a computational tool for constructing numerical modelfor potential flow over an airfoil based on panel methods. Use of the software is illustrated by implementing a specific model using Hess and Smith panel method to compute the flow over a member of the NACA four-digit airfoils.
\end{abstract}

Keywords: Hess and Smith, Laplace equation, panel method, potential flow, NACA fourdigit.

\section{Introduction}

Aerodynamics is a branch of fluid dynamics concerned primarily with the design of vehicles moving through air. In the beginning of ninety, a collection of relatively simple numerical models, known as panel methods, was the primary computational tool for estimating some of the aerodynamic characteristics of airplanes and their components for cruise conditions.

Panel methods are numerical models based on simplifying assumptions about the physics and properties of the air flow over an aircraft. The viscosity of air in the flow field is neglected, and the net effect of viscosity on a wing is summarized by requiring that the flow leaves the sharp trailing edge of the wing smoothly.

The compressibility of air is neglected, and the curl of the velocity field is assumed to be zero (no vortices in the flow field). Under these assumptions, the vector velocity describing the flow field can be represented as the gradient of a scalar potentialvelocity, $\mathbf{V}=\nabla \phi$ and the resulting flow is referred to as potential flow. A statement of 
conservation of mass in the flow field leads to Laplace's equation as the governing equation for the potentialvelocity $\nabla_{\phi}^{2}=0$.

To solve the problem of potential flow over a solid object, Laplace's equation must be solved subject to the boundary condition that there is no flow across the surface of the object. This is usually referred to as the tangent-flow boundary condition. Additionally, the flow far from the object is required to be uniform. The results of solving Laplace's equation subject to tangent-flow boundary conditions provide an approximation of cruise conditions for an airplane.

Using a vector identity, the solution to this linear partial differential equation can be written in terms of an integral over the surface of the object. This boundary integral contains expressions for surface distributions of basic singular solutions to Laplace's equation. A linear combination of relatively simple singular solutions is also a solution to the differential equation. This superposition of simple solutions provides the complexity needed for satisfying boundary conditions for flow over objects of complex geometry. Panel methods are based on this approach. Commonly used singular solutions for panel methods are referred to as source, vortex, and doublet distributions.

The basic solution procedure for panel methods consists of discretizing the surface of the object with flat panels and selecting singularities to be distributed over the panels in a specified manner, but with unknown singularity-strength parameters. Since each singularity is a solution to Laplace's equation, a linear combination of the singular solutions is also a solution. The tangent-flow boundary condition is required to be satisfied at a discrete number of points called collocation points. This process leads to a system of linear algebraic equations to be solved for the unknown singularity-strength parameters.

\section{Panel method}

There are many choices as to how to formulate a panel method (singularity solutions, variation within a panel, singularity strength and distribution, etc.) The simplest and first truly practical method was due to Hess and Smith, Douglas Aircraft, 1966. It is based on a distribution of sources and vortices on the surface of the geometry. In their method

$$
\phi=\phi_{\infty}+\phi_{S}+\phi_{V}
$$

where, $\phi$ is the total potential function and the three components are the potentials corresponding to the free stream, the source distribution, and the vortex distribution. These last two distributions have potentially locally varying strengths $\sigma(s)$ and $\gamma(s)$.

The potentials created by the distribution of sources/sinks and vortices are given by: 


$$
\begin{aligned}
& \phi_{S}=\int \frac{\sigma(s)}{2 \pi} \ln r d s \\
& \phi_{V}=-\int \frac{\gamma(s)}{2 \pi} O d s
\end{aligned}
$$

Figure 1. Airfoil Analysis Nomenclature for Panel Method.

Notice that in these formulae, the integration is to be carried out along the complete surface of the airfoil. Using the superposition principle, any such distribution of sources/sinks and vortices satisfies Laplace's equation, but we will need to find conditions for $\sigma(s)$ and $\gamma(s)$ such that the flow tangency boundary condition and the Kutta condition are satisfied.

Notice that we have multiple options. In theory, we could:

- Use the source strength distribution to satisfy flow tangency and the vortex distribution to satisfy the Kutta condition.

- Use arbitrary combinations of both sources/sinks and vortices to satisfy both boundary conditions simultaneously.

Hess and Smith made the following valid simplification. Take the vortex strength to be constant over the whole airfoil and use the Kutta condition to fix its value, while allowing the source strength to vary from panel to panel. So that, together with the constant vortex distribution, the flow tangency boundary condition is satisfied everywhere.

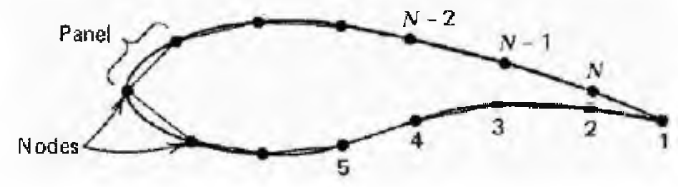

Figure 2. Definition of Nodes and Panels.

We can "discretize" equation (1) in $N$ panels in the following way:

$$
\phi=V_{\infty}(x \cos \alpha+y \sin \alpha)+\sum_{j=1}^{N} \int_{\text {panel }}\left[\frac{\sigma(s)}{2 \pi} \ln r-\frac{\gamma}{2 \pi} \theta\right] d s
$$

Since Equation (3) involves integrations over each discrete panel on the surface of the airfoil, we must somehow parameterize the variation of source and vortex strength within 
each of the panels. Since the vortex strength was considered to be a constant, we only need worry about the source strength distribution within each panel.

Hess and Smith decided to take the simplest possible approximation that is to take the source strength to be constant on each of the panels

$$
\begin{aligned}
& \sigma(s)=\sigma_{i} \text { on panel } i, \\
& i=1, \ldots \ldots ., N
\end{aligned}
$$

Therefore, we have $N+$ lunknown to solve for in our problem: the $N$ panel source strengths $\sigma_{i}$ and the constant vortex strength $\gamma$. Consequently, we will need $N+1$ independent equation which can be obtained byformulating the flow tangency boundary condition at each of the $N$ panels, and by enforcing the Kutta condition. The solution ofthe problem will require the inversion of a matrix of size $(N+1) \times(N+1)$.

\section{Implementation}

Consider the $i$ th panel to be located between the $i$ th and $(i+1)$ th nodes, with its orientation to the $x$-axis given by

$$
\begin{aligned}
& \sin \theta_{i}=\frac{y_{i+1}-y_{i}}{l_{i}} \\
& \cos \theta_{j}=\frac{x_{i+1}-x_{i}}{l_{i}}
\end{aligned}
$$

Where $l_{i}$ is length of the panel. The normal and tangential vectors to this panel, are then given by

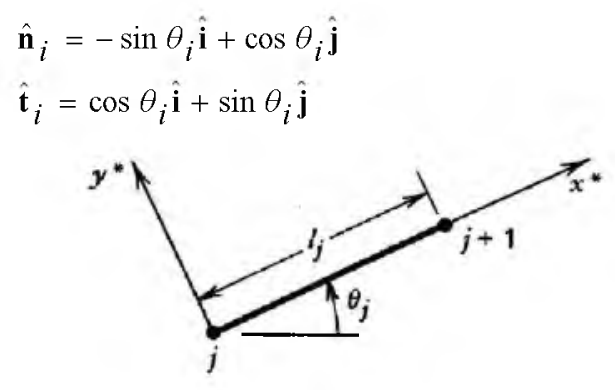

Figure 3. Local Panel Coordinate system.

Furthermore, the coordinates of the midpoint of the panel are given by

$$
\begin{aligned}
& \bar{x}_{i}=\frac{x_{i}+x_{i+1}}{2} \\
& \bar{y}_{i}=\frac{y_{i}+y_{i+1}}{2}
\end{aligned}
$$

And the velocity components at these midpoints are given by 


$$
\begin{aligned}
& u_{i}=u\left(\bar{x}_{i}, \bar{y}_{i}\right) \\
& \bar{v}_{i}=v\left(\bar{x}_{i}, \bar{y}_{i}\right)
\end{aligned}
$$

The flow tangency boundary condition can then be simply written as $(\overrightarrow{\mathbf{u}} \cdot \overrightarrow{\mathbf{n}})=0$, or, for each panel

$$
-u_{i} \sin \theta_{i}+v_{i} \cos \theta_{i}=0 \quad \text { for } i=1, \ldots, N
$$

While the Kutta condition is simply given by

$$
u_{1} \cos \theta_{1}+v_{1} \sin \theta_{1}=-u_{N} \cos \theta_{N}-v_{N} \sin \theta_{N}
$$

Now, the velocity at the midpoint of each panel can be computed by superposition of the contributions of all sources and vortices located at the midpoint of everypanel (including itself). Since the velocity induced by the source or vortex on a panel is proportional to the source or vortex strength in that panel, $\sigma_{i}$ and $\gamma$ can be pulled out of the integral in equation (3) to yield

$$
\begin{aligned}
& u_{i}=V_{\infty} \cos \alpha+\sum_{j=1}^{N} \sigma_{j} u_{s i j}+\gamma \sum_{j=1}^{N} u_{v i j} \\
& v_{i}=V_{\infty} \sin \alpha+\sum_{j=1}^{N} \sigma_{j} v_{s i j}+\gamma \sum_{j=1}^{N} v_{v i j}
\end{aligned}
$$

Where $u_{s i j}, v_{s i j}$ are the velocity components at the midpoint of panel $i$ induced by a source of unit strength at the midpoint of panel $j$. A similarinterpretation can be found for $u_{v i j}, v_{v i j}$. In a coordinate system tangentialand normal to the panel, we can perform the integrals in equation (3) bynoticing that the local velocity components can be expanded into absoluteones according to the following transformation:

$$
\begin{aligned}
& u=u^{*} \cos \theta_{j}-v^{*} \sin \theta_{j} \\
& v=u^{*} \sin \theta_{j}-v^{*} \cos \theta_{j}
\end{aligned}
$$

Now, the local velocity component at the midpoint of the $i$ th panel due to a unitstrength source distribution on this $j$ th panel can be written as

$$
\begin{aligned}
& u_{s i j}^{*}=\frac{1}{2 \pi} \int_{0}^{l} \frac{x^{*}-t}{\left(x^{*}-t\right)^{2}+y^{* 2}} d t \\
& v_{s i j}^{*}=\frac{1}{2 \pi} \int_{0}^{l} \frac{y^{*}}{\left(x^{*}-t\right)^{2}+y^{* 2}} d t
\end{aligned}
$$

Where $x^{*}, y^{*}$ are the coordinates of the midpoint of panel $i$ in the local coordinate system of panel $j$, carrying out the integrals in equation (7) we find that 


$$
\begin{aligned}
& u_{s i j}^{*}=\left.\frac{-1}{2 \pi} \ln \left[\left(x^{*}-t\right)^{2}+y^{* 2^{-}}\right]^{\frac{1}{2}}\right|_{t=0} ^{t=l} j \\
& v_{s i j}^{*}=\left.\frac{1}{2 \pi} \tan ^{-1} \frac{y^{*}}{x^{*}-t}\right|_{t=0} ^{t=l} j
\end{aligned}
$$

These results have a simple geometric interpretation that can be discerned by looking at the figure 4 below. One can say that

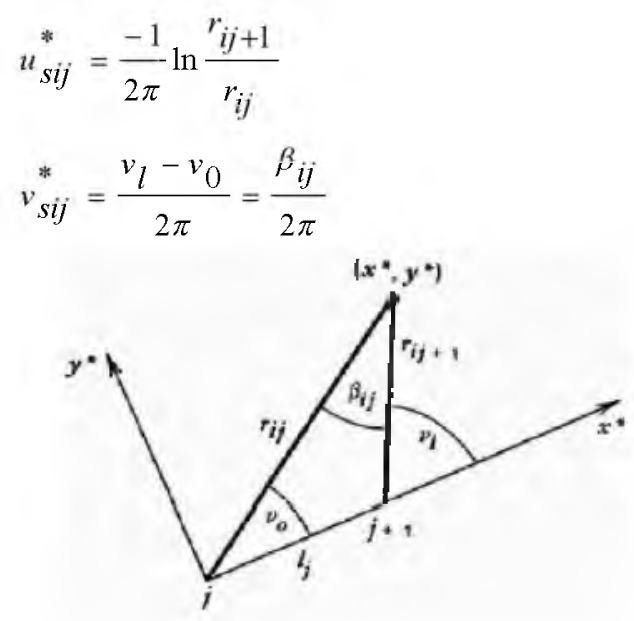

Figure 4. Geometric Interpretation of Source and Vortex Induced Velocities.

Ther $r_{i j}$ is the distance from the midpoint of panel $i$ to the $j$ th node, while $\beta_{i j}$ is the angle subtended by the $j$ th panel at the midpoint of panel $i$. Notice that $u_{s i}^{*}=0$, but the value of $v_{s i i}^{*}$ is not so clear. When the point of interest approaches the midpoint of the panel from the outside of the airfoil, this angle, $\beta_{i i} \rightarrow \pi$. However, when the midpoint of the panel is approached from the inside of the airfoil, $\beta_{i i} \rightarrow-\pi$. Since we are interested in the flow outside of the airfoil only, we will always take $\beta_{i i}=\pi$.

Similarly, for the velocity field induced by the vortex on panel $j$ at the midpoint of panel $i$ we can simply see that

$$
\begin{aligned}
& u_{v i j}^{*}=-\frac{1}{2 \pi} \int_{0}^{l_{j}} \frac{y^{*}}{\left(x^{*}-t\right)^{2}+y^{* 2}} d t=\frac{\beta_{i j}}{2 \pi} \\
& v_{v i j}^{*}=-\frac{1}{2 \pi} \int_{0}^{l} \frac{x^{*}-t}{\left(x^{*}-t\right)^{2}+y^{* 2}} d t=\frac{1}{2 \pi} \ln \frac{r_{i j+1}}{r_{i j}}
\end{aligned}
$$

And finally, the flow tangency boundary condition, using equation (5), and undoing the local coordinate transformation of equation (6) can be written as 


$$
\sum_{j=1}^{N} A_{i j} \sigma_{j}+A_{i N+\mathrm{l} \gamma}=b_{i}
$$

Where,

$$
\begin{aligned}
& A_{l j}=-u_{s i j} \sin \theta_{i}+v_{s i j} \cos \theta_{i} \\
&=-u_{s i j}^{*}\left(\cos \theta_{j} \sin \theta_{i}-\sin \theta_{j} \cos \theta_{i}\right) \\
&+v_{s i j}^{*}\left(\sin \theta_{j} \sin \theta_{i}+\cos \theta_{j} \cos \theta_{i}\right)
\end{aligned}
$$

This yield

$$
2 \pi A_{i j}=\sin \left(\theta_{i}-\theta_{j}\right) \ln \frac{r_{i j+1}}{r_{i j}}+\cos \left(\theta_{i}-\theta_{j}\right) \beta_{i j}
$$

Similarly for the vortex strength coefficient

$$
2 \pi A_{i N+1}=\sum_{j=1}^{N} \cos \left(\theta_{i}-\theta_{j}\right) \ln \frac{r_{i j+1}}{r_{i j}}+\sin \left(\theta_{i}-\theta_{j}\right) \beta_{i j}
$$

The right hand side of this matrix equation is given by

$$
b_{i}=V_{\infty} \sin \left(\theta_{i}-\alpha\right)
$$

The flow tangency boundary condition gives us $N$ equations. We need an additional one provided by the Kutta condition in order to obtain a system that can be solved. According to equation (4)

$$
\sum_{j=1}^{N} A_{N+1, j} \sigma_{j}+A_{N+1, N+1} \gamma=b_{N+1}
$$

After similar manipulations we find that

$$
\begin{aligned}
2 \pi A_{N+1, j} & =\sum_{k=1, N} \sin \left(\theta_{k}-\theta_{j}\right) \beta_{k j}-\cos \left(\theta_{k}-\theta_{j}\right) \ln \frac{r_{k j+1}}{r_{k j}} \\
2 \pi A_{N+1, N+1} & =\sum_{k=1, N} \sum_{j=1}^{N} \sin \left(\theta_{k}-\theta_{j}\right) \ln \frac{r_{k j+1}}{r_{k j}}+\cos \left(\theta_{k}-\theta_{j}\right) \beta_{k j} \\
b_{N+1} & =-V_{\infty} \cos \left(\theta_{k}-\alpha\right)-V_{\infty} \cos \left(\theta_{N}-\alpha\right)
\end{aligned}
$$

Where the sums $\sum_{k, N}$ are carried out only over the first and last panels and not the range [1:

$N]$. These various expressions set up a matrix problem of the kind

$$
[\mathrm{A}] \cdot\{\mathrm{x}\}=\{\mathrm{b}\}
$$

Where the size of matrix $A$ is $(N+1) \times(N+1)$. This system can be sketched as follows: 
Buyung Junaidin

$$
\left[\begin{array}{cccccc}
A_{11} & \cdots & A_{1 i} & \cdots & A_{1 N} & A_{1, N+1} \\
\vdots & & \vdots & & \vdots & \vdots \\
A_{i 1} & \cdots & A_{i i} & \cdots & A_{i N} & A_{i, N+1} \\
\vdots & & \vdots & & \vdots & \vdots \\
A_{N 1} & \cdots & A_{N i} & \cdots & A_{N N} & A_{N, N+1} \\
A_{N+1, N+1} & \cdots & A_{N+1, N+1} & \cdots & A_{N+1, N+1} & A_{N+1, N+1}
\end{array}\right]\left[\begin{array}{c}
\sigma_{1} \\
\vdots \\
\sigma_{i} \\
\vdots \\
\sigma_{N} \\
\gamma
\end{array}\right]=\left[\begin{array}{c}
b_{1} \\
\vdots \\
b_{i} \\
\vdots \\
b_{N} \\
b_{N+1}
\end{array}\right]
$$

Finally,we have solved the system for the unknowns of the problem; it is easy to construct the tangential velocity at the midpoint of each panel according to the following formula

$$
\begin{aligned}
V_{t i}= & V_{\infty} \cos \left(\theta_{i}-\alpha\right)+\sum_{j=1}^{N} \frac{\sigma_{j}}{2 \pi}\left[\sin \left(\theta_{i}-\theta_{j}\right) \beta_{i j}-\cos \left(\theta_{i}-\theta_{j}\right) \ln \frac{r_{i j+1}}{r_{i j}}\right] \\
& +\frac{\gamma}{2 \pi} \sum_{j=1}^{N}\left[\sin \left(\theta_{i}-\theta_{j}\right) \ln \frac{r_{i j+1}}{r_{i j}}+\cos \left(\theta_{i}-\theta_{j}\right) \beta_{i j}\right]
\end{aligned}
$$

And knowing the tangential velocity component, we can compute the pressure coefficient at the midpoint of each panel according to the following formula

$$
C_{p}\left(\bar{x}_{i}, \bar{y}_{i}\right)=1-\frac{V_{t i}^{2}}{V_{\infty}^{2}}
$$

From this equation (15) the force coefficientscan be computed.

$$
\begin{aligned}
& c_{l}=c_{y} \cos \alpha-c_{x} \sin \alpha \\
& c_{d}=c_{y} \sin \alpha+c_{x} \cos \alpha
\end{aligned}
$$

Where,

$$
\begin{aligned}
& c_{x}=\oint_{p} d x \\
& c_{y}=\oint_{p} d y
\end{aligned}
$$

In this paper we use a computational tool (MATLAB code)to compute the geometry of NACA 0012 airfoil and constructing the numerical models to compute aerodynamics characteristic of airfoil with variation of angle of attack $\alpha=1^{0}, 2^{0}, 3^{0}, 4^{0}, 5^{0}$.

\section{Result}

The computational tool gives us good results as compared to the experimental results acquired for NACA 0012 airfoil and with other existing simulation results (XFOIL, panel2d, etc).

Figures 5 represent the geometries approximation of NACA 0012 airfoil for the twodimensional segmentation (panels).

Figures $6,7,8$ and 9 represent the distribution of local pressure coefficient and the pressure difference obtained between the wing upper and lower parts along the chord. At high 
angle of attack the curves show that the pressure difference reaches very high values at the leading edge and quasi-null value at the trailing edge, thereby satisfying the KUTTA condition.

Table 1 shows, the aerodynamics characteristic obtained from panel method with the assumption incompressible and inviscid flow (effect of viscosity is neglected), the entire characteristic increases when the angles of attack increase.

Table 2 shows, the experiment result of aerodynamics characteristic with condition incompressible and viscous flow $\left(\operatorname{Re}=7 \times 10^{5}\right)$. The lift coefficient from panel method and experiment result is similar, but for the drag coefficient it's different because we use assumption for panel method that the flow is inviscid.

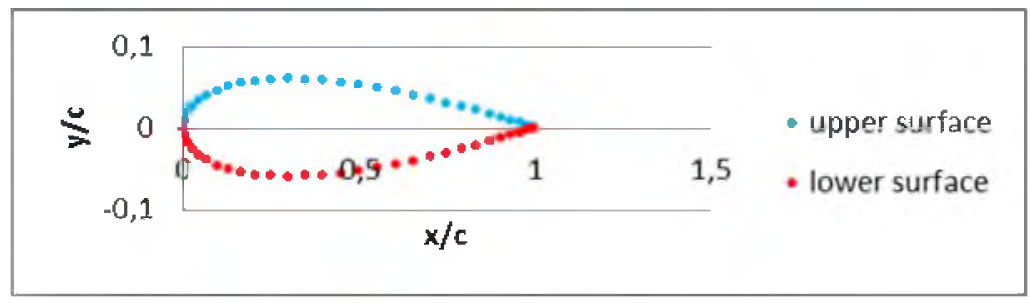

Figure 5. NACA 0012 Airfoil Geometry.

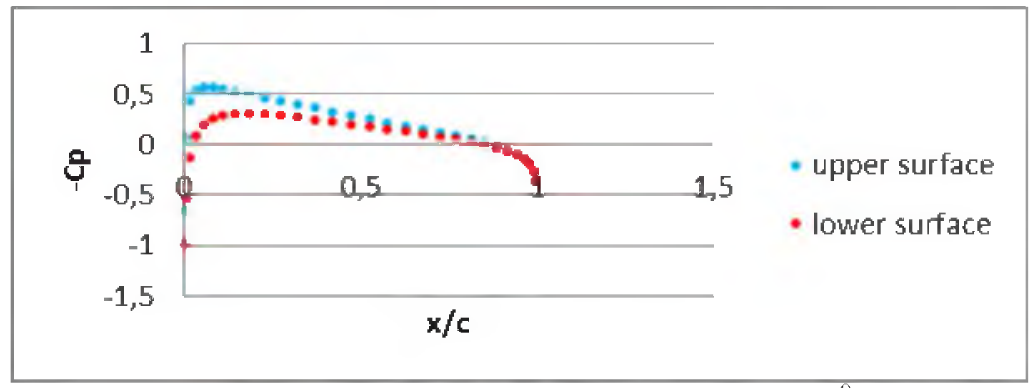

Figure 6. Pressure coefficient distribution for NACA 0012, $\alpha=1^{0}, 60$ panels.

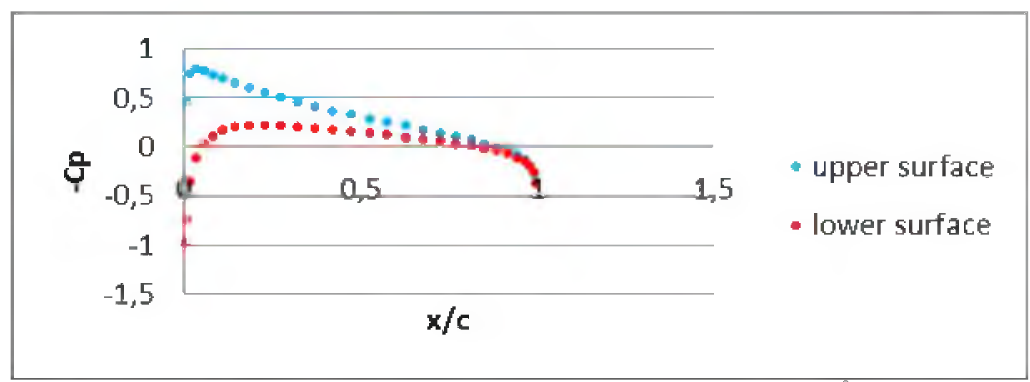

Figure 7. Pressure coefficient distribution for NACA $0012, \alpha=2^{0}, 60$ panels. 


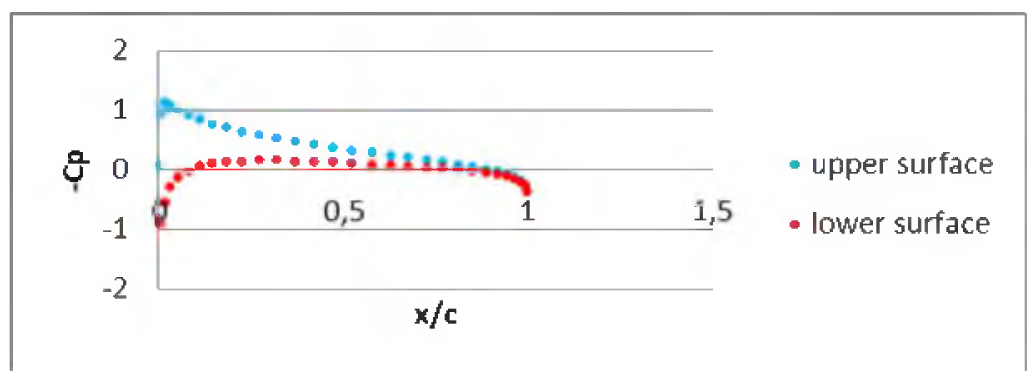

Figure 8. Pressure coefficient distribution for NACA 0012, $\alpha=3^{0}, 60$ panels.

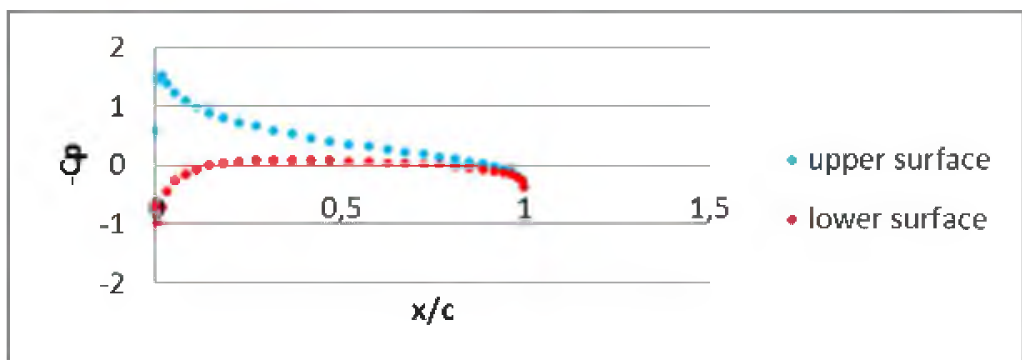

Figure 9. Pressure coefficient distribution for NACA 0012, $\alpha=4^{0}, 60$ panels.

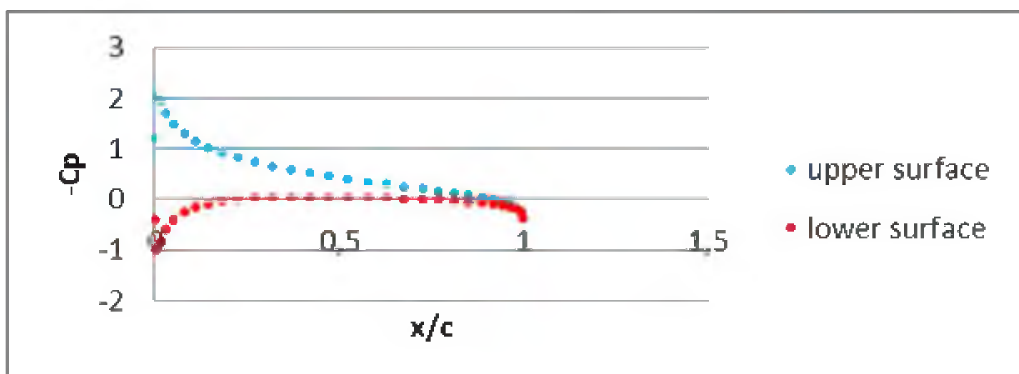

Figure 10. Pressure coefficient distribution for NACA 0012, $\alpha=5^{0}, 60$ panels.

Table 1. Panel Method result of NACA 0012 Airfoil.

\begin{tabular}{ccc}
\hline $\begin{array}{c}\boldsymbol{\alpha} \\
\text { (degre } \\
\text { e) }\end{array}$ & $\mathbf{C}_{\mathbf{l}}$ & $\mathbf{C}_{\mathbf{d}}$ \\
\hline 1 & 0.11845 & 0.00066 \\
2 & 0.23861 & 0.00068 \\
3 & 0.35869 & 0.00071 \\
4 & 0.47865 & 0.00075 \\
5 & 0.59847 & 0.00081 \\
\hline
\end{tabular}

Table 2. Experiment Result of NACA 0012 Airfoil.

\begin{tabular}{ccc}
\hline $\begin{array}{c}\text { o. } \\
\text { degre } \\
\mathbf{e})\end{array}$ & $\mathbf{C}_{\mathbf{l}}$ & $\mathbf{C}_{\mathbf{d}}$ \\
\hline 1 & 0.11 & 0.0133 \\
2 & 0.22 & 0.0134 \\
3 & 0.33 & 0.0138 \\
4 & 0.44 & 0.0145 \\
5 & 0.55 & 0.0155 \\
\hline
\end{tabular}




\section{Conclusions}

The method based on the singularity distribution (sources, sinks, doublets, and vortices) finds a largeapplication in the resolution of problems encountered by aero plane designers, in particular during the analysis phase. It was used in this paper to compute the aerodynamic characteristics of the NACA four digit families. The developed program is based on the solution of the integral equation. It provides inparticular the velocity and pressure fields as well as the local and total forces of the studiedconfigurations.

For a two-dimensional flow, the proposed model is based on the representation of the realobstacle by a solid composed of many plane facets. On each one, is disposed a set of singularities thechoice of which type is always linked to the type of flow phenomenon on these facets.

In the perspective it is very possible to deepen the present research by the insertion of theviscous effects due to the boundary layer and by the resolution of the aero elastic problem. 


\section{References}

Anderson, John D., 1991, Fundamental of Aerodynamics, $2^{\text {nd }}$ ed., McGraw-Hill, Inc., Singapore.

Applied Computational Aerodynamics, Virginia Tech, online: http://www.dept.aoe.vt.edu/ mason/Mason_f/CAtxtChap4.pdf, (1998, February 24).

G. Dimitriadis, Panel Method, Universite de Liege, online: http://www.ltasaea.ulg. ac. be/cms/uploads/Aerodynamics04.pdf.

Hess-Smith Panel method, Universidad Politécnica de Madrid, online: http://canal. etsin. upm.es/CFDWORKSHOP/lect3-4.pdf, (2005, January 3).

J. Katz \& A. Plotkin., 1991, Low-Speed Aerodynamics from Wing theory to Panel Methods, McGraw-Hill, Inc., Singapore.

Moran, J., 1984, An Introduction to Theoretical and Computational Aerodynamics, John Wiley \& Sons, New York. 